\title{
Mitos de violación, creencias que justifican la violencia sexual: una revisión sistemática
}

\section{Rape myths, beliefs that justify sexual violence: \\ a systematic review \\ Mitos do estupro, crenças que justificam a violência sexual: uma revisão sistemática}

Fecha de recepción: 2020/0I/27 | Fecha de evaluación: 2020/06/10 | Fecha de aprobación: 2020/09/22

$\begin{array}{rr}\text { Carol Murray } & \text { Carlos Calderón } \\ \text { Magíster en Educación Mención Curriculum } & \text { Doctor en Metodología de las Ciencias del Comportamiento y de la Salud } \\ \text { Escuela de Psicología, Universidad Católica del Norte } & \text { Académico, Escuela de Psicología } \\ \text { Antofagasta, Chile } & \text { Universidad Católica del Norte } \\ \text { cassjomu@gmail.com } & \text { ccalderon@ucn.cl }\end{array}$

Para citar este artículo / To reference this article / Para citar este artigo: Murray, C. \& Calderón, C. (2021). Mitos de violación, creencias que justifican la violencia sexual: una revisión sistemática. Revista Criminalidad, 63(2), I I5-I 30. https://doi.org// 0.4774I//7943 I08.320

\section{Resumen}

En investigación social existe una importante línea de estudios que intentan comprender el fenómeno de la violencia sexual. Un constructo que ha centrado el interés de no pocos investigadores corresponde al de "mitos de violación". Este concepto agrupa creencias erróneas respecto a la atribución de culpabilidad del agresor y la responsabilidad de la víctima en caso de violación sexual. El objetivo del presente artículo fue realizar una revisión sistemática de los estudios empíricos que reportan evidencia acerca de la relación entre la aceptación de mitos de violación y variables psicosociales. Las bases de datos consultadas fueron, ProQuest, Web of Science, Scopus, PsycINFO, EBSCOhost y JSTOR. Se seleccionaron estudios empíricos en español e inglés publicados entre los años 2009 y 2019 . Se encontraron 96 artículos que cumplían con los criterios de selección. Dentro de los resultados el 63,4\% de las investigaciones se desarrollaron en Estados Unidos, 24,7\% en países de Europa y solo I \% en América Latina. Los hallazgos muestran que los hombres son los que mantienen mayores niveles de aceptación de los mitos de violación en la mayoría de los estudios. Además, se encontraron relaciones con variables como violencia interpersonal y violencia sexual, rasgos de personalidad, variables ideológicas, psicosociales, sexuales y de género. Finalmente se discute sus implicancias y limitaciones.

\section{Palabras clave}

Violencia sexual (fuente: Tesauro Criminológico - Instituto Interregional de las Naciones Unidas para Investigaciones sobre la Delincuencia y la Justicia - UNICRI). Atribución, mitos de violación, género, revisión sistemática (fuente: autores). 


\section{Abstract}

In social research, there is an important line of studies that try to understand the phenomenon of sexual violence. A construct that has focused the interest of not a few researchers corresponds to that of "rape myths". This concept brings together a set of erroneous beliefs regarding the attribution of guilt of the aggressor and the responsibility of the victim in case of rape. The objective of this study was to conduct a systematic review of empirical studies that have reported evidence about the relationship between the acceptance of rape myths and psychosocial variables. The databases consulted were, ProQuest, Web of Science, Scopus, PsycINFO, EBSCOhost, JSTOR.
We selected empirical studies in Spanish and English that have been published between 2009 and 2019. We found 96 articles that met the selection criteria. Within the results, $63.4 \%$ of the studies have been developed in the United States, $24.7 \%$ in European countries and only one in Latin America (1.0\%). The findings show that men maintain the highest levels of acceptance of rape myths in most studies. In addition, relationships have been found with variables such as interpersonal violence and sexual violence, personality traits, ideological, psychosocial, sexual and gender variables. Finally, its implications and limitations are discussed.

\section{Keywords}

Sexual violence. (source: Criminological Thesaurus - United Nations Interregional Crime and Justice Research Institute UNICRI). Attribution, rape myths, gender, systematic review. (source: author).

\section{Resumo}

Na pesquisa social, há uma importante linha de estudos que tentam entender o fenômeno da violência sexual. Uma construção que tem focado o interesse de poucos pesquisadores corresponde à de "mitos do estupro". Esse conceito reúne um conjunto de crenças errôneas sobre a atribuição de culpa do agressor e a responsabilidade da vítima em caso de estupro. O objetivo deste estudo foi realizar uma revisão sistemática de estudos empíricos que relataram evidências sobre a relação entre a aceitação de mitos de estupro e variáveis psicossociais. As bases de dados consultadas foram: ProQuest, Web of Science, Scopus, PsycINFO, EBSCOhost, JSTOR. Selecionamos estudos empíricos em espanhol e inglês que foram publicados entre 2009 e 2019. Encontramos 96 artigos que atenderam aos critérios de seleção. Dentro dos resultados, 63,4\% dos estudos foram desenvolvidos nos Estados Unidos, 24,7\% em países europeus e apenas um na América Latina ( $1,0 \%)$. Os achados mostram que os homens mantêm os mais altos níveis de aceitação dos mitos do estupro na maioria dos estudos. Além disso, têm sido encontradas relações com variáveis como violência interpessoal e violência sexual, traços de personalidade, variáveis ideológicas, psicossociais, sexuais e de gênero. Finalmente, suas implicações e limitações são discutidas.

\section{Palavras-chave}

Violência sexual. (fonte: Thesaurus Criminológico - Instituto Inter-regional de Pesquisa em Crime e Justiça das Nações Unidas - UNICRI). Atribuição, mitos do estupro, gênero, revisão sistemática. (fonte: autor).

\section{Introducción}

La violencia sexual es considerada una de las problemáticas más graves que deben enfrentar los Estados, tanto por la necesidad de garantizar acceso a la justicia protegiendo los derechos humanos del (la) afectado(a) y del (la) agresor(a), como por las consecuencias en la salud sexual, reproductiva y mental en la víctima (Comisión Interamericana de Derechos Humanos, 201 I; Organización Panamericana de Salud 
[OPS], 2003). Es una problemática mundial, pues todos los países reportan violencia sexual en algún porcentaje, la que sufren principalmente mujeres y otros grupos vulnerables como niños, niñas, adolescentes y personas con discapacidad (OPS, 2003).

Según datos de la Organización Mundial de la Salud, el $30 \%$ de las mujeres mayores de 15 años experimenta violencia física y sexual por parte de sus parejas, frente al $7,2 \%$ que han sido violentadas sexualmente fuera del contexto de pareja. Cifra que se eleva al 10,7\% en Latinoamérica y el Caribe (García-Moreno et al., 20l3). Estos datos revelan que, a diferencia de la creencia general, la mayor proporción de violencia sexual sucede al interior de la pareja.

En Colombia, el Grupo Centro de Referencia Nacional sobre Violencia del Instituto Nacional de Medicina Legal y Ciencias Forenses (INMLCF) reportó 26.065 exámenes médico-legales por presuntos casos de delitos sexuales durante el año 2018, lo que correspondió a un aumento de 2.267 casos con respecto al año 2017 (INMLCF, 2019). Según las estadísticas del Ministerio Público de Chile (2019) entre enero y septiembre del 2019 se registraron 26.577 víctimas ingresadas por delitos sexuales. Una encuesta realizada en Chile a estudiantes universitarios muestra que la tasa de victimización en las mujeres llega al $52 \%$ y en hombres al $48 \%$ (Schuster et al., 2016). En Argentina según la Unidad Fiscal Especializada en Violencia contra las Mujeres, del Ministerio Público Fiscal (2019), durante el 2018 las denuncias presentadas por delitos sexuales alcanzaron 16.974 víctimas en todo el país.

Debido a las graves consecuencias que genera la violencia sexual social e individualmente (Elliot et al., 2004; Kaukinen \& DeMaris, 2005), el estudio de esta problemática ha tomado relevancia en las últimas décadas, transformándose en un tema de interés tanto para los académicos, como para otros grupos sociales (Contreras et al., 2016; Romero-Sánchez, 20I2).

Dentro de las áreas de estudios que han ayudado a visibilizar este tipo de violencia encontramos: las teorías feministas que exponen, problematizan y cuestionan los discursos hegemónicos y dominantes presentes en las narrativas sociales, los que promueven estereotipos de víctimas y victimarios que sesgan y justifican la violencia sexual y de género (Millares, 2020; Payne et al., 1999); la criminología que busca generar evidencia de las características sociales e individuales de los agresores y las posibles causas que llevan a los sujetos a cometer actos que atentan contra las libertades individuales; y la victimología que ha relevado los procesos de victimización y las consecuencias a corto y largo plazo en las personas y grupos que han sido expuestos a los distintos tipos de violencia (Da Silva et al., 20I8; Fattah, 2014).

Una de las conceptualizaciones que intentan explicar cómo se legitima y valida la violencia sexual son las creencias erróneas respecto de la atribución de responsabilidad de la víctima y la culpabilidad del agresor frente a un hecho de agresión sexual; estas creencias son conocidas como mitos de violación (Burt, 1980; Payne et al., 1999). El primer acercamiento al concepto de mitos de violación fue durante la década de los setenta. Los mitos de violación se definen como actitudes y creencias culturalmente arraigadas, falsas, amplias y persistentes, que tienen como función justificar, negar o trivializar las agresiones sexuales que los hombres cometen en contra de las mujeres (Grubb \& Turner, 2012; Javaid, 20I5; Maxwell \& Scott, 20I4).

El papel que desempeñan estas creencias resulta relevante para explicar la respuesta social frente a la violencia sexual y cómo esta influye en las atribuciones de responsabilidad del agresor y la víctima (Hine \& Murphy, 2018); la comprensión del comportamiento de los victimarios, y conocer la etiología de los actos de agresión sexual (Debowska et al., 2016); y entender la reacción de la víctima con relación a su estado emocional y cómo estas creencias afectan la decisión de realizar una denuncia (Wilson et al., 2017).

Con base en la evidencia acumulada, los mitos de violación pueden ser agrupados en cuatro tipos generales (Gerger et al., 2007; Payne et al., 1999): (a) culpar a la víctima; se soporta en la idea de que la mujer tiene deseos inconscientes de ser violada, lo cual se manifiesta en apariencia y conductas provocadoras; (b) incredulidad en las denuncias de violación; se centran en creencias de que las víctimas de violación exageran lo ocurrido, que las acusaciones son falsas y que lo que se busca con la denuncia son ganancias secundarias; (c) mitos que exoneran al perpetrador; afirman que los hombres tienen mayor deseo sexual, que es incontrolable, y la idea de que los violadores tienen deseos sexuales excesivos, y (d) la creencia de que solo algunos tipos de mujeres son violadas; por ejemplo, las mujeres que se visten con ropa provocativa, las que se exponen asistiendo a ciertos lugares.

Entre los mitos más estudiados que se asocian al último grupo encontramos el consumo de alcohol y la vestimenta de la víctima. Estos mitos específicos muestran tener gran valor predictivo con respecto a la aceptación de los mitos de violación. Además, estos mitos son los que ejercen un efecto directo en la 
atribución deresponsabilidadala víctimayla disminución de culpabilidad del agresor (Romero-Sánchez et al., 2017; Süssenbach et al., 2015; Venema, 2016).

Si bien se registran algunos estudios teóricos y sistematizaciones que dan cuenta de las relaciones de los mitos de violación con variables psicosociales, hasta la fecha ningún artículo ha hecho una revisión que sintetice y agrupe de manera sistemática todas las investigaciones empíricas que han involucrado la aceptación de mitos de violación (Grubb \& Turner, 20I2; Javaid, 20I5; Maxwell \& Scott, 20I4). El objetivo del presente estudio teórico es efectuar una revisión sistemática de las investigaciones empíricas existentes hasta la fecha que han estudiado el constructo mitos de violación y las relaciones con otras variables entre los años 2009 y 2019.

\section{Método}

\section{Procedimiento y estrategias de búsqueda}

Se realizó una revisión sistemática de la literatura siguiendo los lineamientos y recomendaciones de la declaración Prisma (Liberati et al., 2009). Se configuró la búsqueda de artículos publicados entre los años 2009 y 2019 consultando seis bases de datos $(n=733$; ProQuest $=122$, Web of Science $=223$, Scopus $=91$, PsycINFO $=50$, EBSCOhost $=98$, JSTOR $=149) . \mathrm{La}$ búsqueda de artículos se limitó a estudios en inglés y español utilizando las palabras clave "aceptación de mitos de violación" y "rape myth acceptance". La indagación se acotó a las siguientes áreas de investigación: social psychology; social sciences y social behavior. Se incluyeron solo artículos que aportasen datos empíricos, descartando trabajos teóricos sobre mitos de violación.

\section{Selección de los estudios}

Los estudios se seleccionaron por etapas (véase Figura I). Primero, se compilaron todos los artículos recolectados en las bases de datos antes señaladas $(n=738)$. Segundo, se leyeron los títulos y se eliminaron todos los artículos duplicados $(n=220)$. Tercero, se procedió a la lectura de títulos, resúmenes, palabras clave e instrumentos utilizados en cada artículo, eliminando los documentos que no cumplían con los criterios de inclusión, los que respondían a estudios

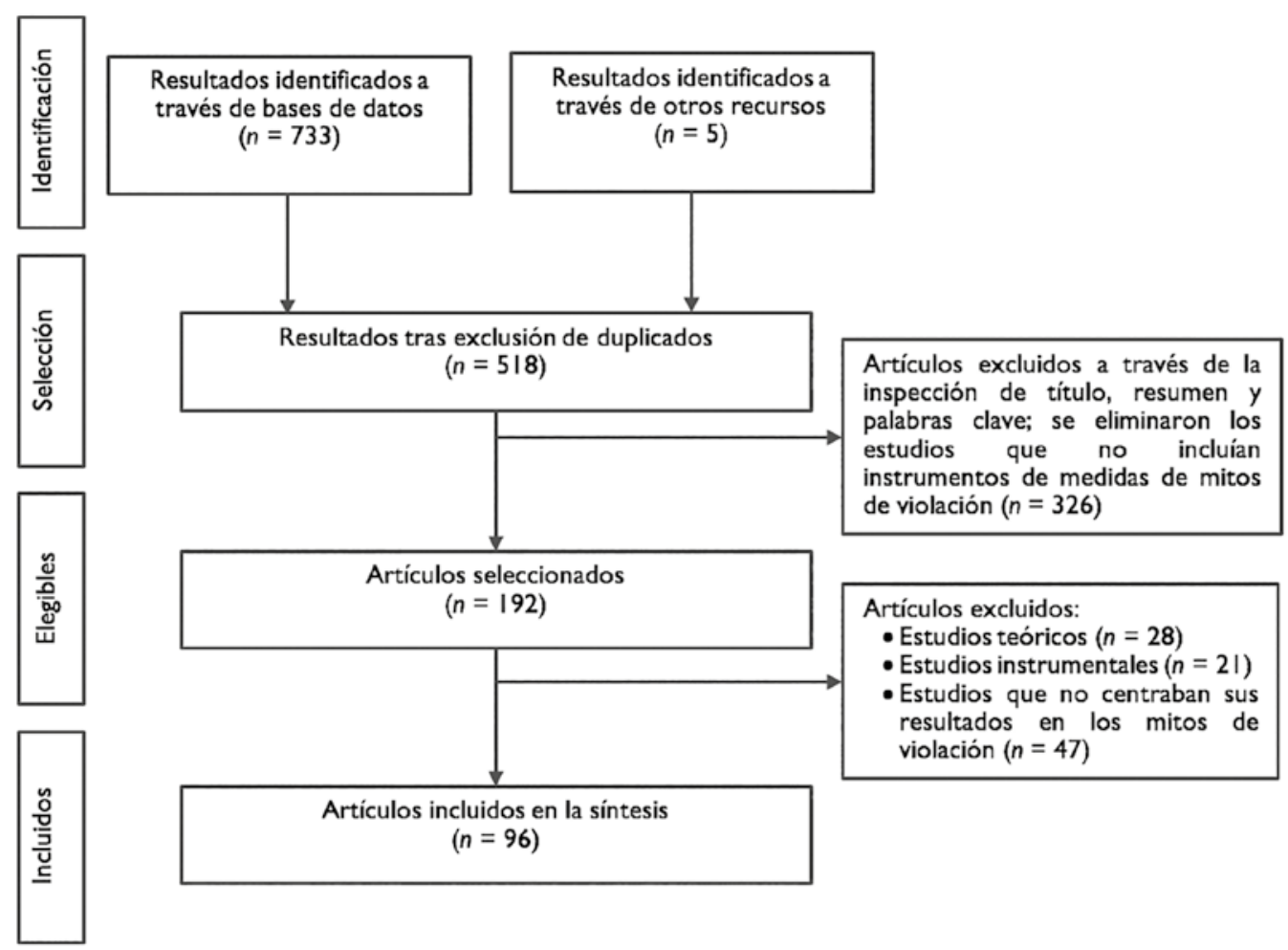

Figura I. Proceso de selección de artículos.

Fuente: elaboración propia. 
ejecutados con metodologías cualitativas y sin medidas cuantificables de los mitos de violación $(n=326)$. Finalmente, se llevó a cabo la lectura de los textos eliminando los artículos sobre estudios teóricos, instrumentales o que sus resultados no se centraban en la aceptación de mitos de violación $(n=96)$. Se puso especial atención a los estudios que desarrollaron intervenciones de prevención y educación en violencia sexual asociada a los mitos de violación.

Una vez finalizada la selección $(n=96)$, se procedió a la síntesis de resultados con el fin de comparar los diferentes estudios. Este procedimiento se efectuó por medio de la extracción de los siguientes datos: (a) autor(es) y año de publicación, (b) muestra(s), (c) país donde se desarrolló la investigación, (d) diseño de investigación, (e) instrumento de medida de aceptación de mitos de violación, (f) variables/manipulaciones incluidas en los estudios y $(\mathrm{g})$ principales resultados.

\section{Resultados}

La presentación de los resultados se divide en dos secciones: (a) características descriptivas de los estudios y (b) resultados de la relación de los mitos de violación con otras variables.

\section{Caracterización descriptiva de los estudios}

La Tabla I muestra los porcentajes según algunas características de los 96 artículos incluidos en la síntesis.

En cuanto a los participantes, del total de estudios, participaron 122 muestras distintas. De ellas, el $66,4 \%(n=81)$ fueron universitarios, $17,2 \%$ $(n=21)$ población general, $6,6 \%(n=8)$ agentes de policía y 9,8\% $(n=12)$ otros tipos de población (p. ej. prisioneros hombres, víctimas). Respecto al país donde se desarrollaron las investigaciones, de las 96 publicaciones, se identificaron a 17 países distintos en los estudios, siendo los más frecuentes Estados Unidos con un 63,4\% $(n=64)$, Reino Unido con un $13,9 \%(n=14)$, Alemania con un 4,9\% $(n=5)$, España con un $2,9 \%(n=3)$, India con un $2,9 \%(n=3)$ y otros países (p. ej. Canadá, Corea del Sur, Indonesia, Italia, Israel, Japón, México, Namibia, Polonia, Sudáfrica) con un II,8\%, $(n=12)$, algunos estudios se ejecutaron en

\section{Tabla I.}

\section{Porcentajedeestudios por tipo de muestra, país, diseño del estudio y tipo de instrumento utilizado}

\begin{tabular}{|c|c|c|c|}
\hline Tipos de muestra & Porcentajes & Países & Porcentajes \\
\hline Agentes de policía & 6,6 & Otros países & 11,8 \\
\hline Estudiantes universitarios & 66,4 & Reino Unido & 13,9 \\
\hline Otros & 4,9 & EE. UU. & 63,4 \\
\hline Población general & 17,2 & Alemania & 4,9 \\
\hline Prisioneros varones & 1,6 & España & 2,9 \\
\hline Víctimas & 3,3 & India & 2,9 \\
\hline Tipos de estudios & Porcentajes & Instrumentos & Porcentajes \\
\hline Correlacionales & 69,8 & IRMAS & 40,8 \\
\hline Cuasiexperimentales & 22,9 & UIRMA & 16,5 \\
\hline Descriptivos & 5,2 & AMMSA & 11,7 \\
\hline Exploratorios & 2,1 & RMAS & 15,5 \\
\hline
\end{tabular}

Fuente: elaboración propia. 
más de un país. Respecto a los tipos de diseños de los estudios, de los 96 artículos, 67 (68,6\%) fueron correlacionales, $22(22,8 \%)$ cuasiexperimentales, 5 (6\%) descriptivos y $2(2,4 \%)$ exploratorios.

Con relación a los instrumentos de medida de mitos de violación, del total de artículos revisados 42 utilizaron la escala de aceptación de mitos de violación de Illinois Irma (Payne et al.,1999), 17 la escala actualizada de aceptación del mito de la violación de Illinois UIRMA (McMahon \& Farmer, 20II), 16 la escala de mitos de violación de Burt RMAS (Burt, 1980), 12 emplearon la escala de mitos modernos de violación AMMSA (Gerger et al., 2007), 4 la escala de aceptación de mitos de violación masculina MRMS (Melanson, 1998), 8 usaron otros instrumentos, como el KRMAS-R (Oh \& Neville, 2004); RMA de Ward (1988); AMV de Saldívar et al. (2004); mitos de violación de Burgess (2007) y SRS (StruckmanJohnson \& Struckman-Johnson, 1992). Por último, 4 aplicaron medidas creadas por los autores (Fox \& Cook, 20I I; Hockett et al., 2016; Thompson \& Ortiz, 2016; Zinzow et al., 2018).

Las variables que se han relacionado con los mitos de violación con mayor frecuencia son aquellas vinculadas a contenidos ideológicos como: sexismo, autoritarismo de ala de derecha, dominancia social; y variables vinculadas a la sexualidad y género. Otras variables incluidas que también presentan alta presencia en los estudios son las relativas a constructos psicosociales (p. ej. actitud hacia la violencia sexual, actitud hacia la policía) y finalmente en menor medida las variables psicológicas individuales (p. ej. rasgos de personalidad, autoestima).

Del total de investigaciones cuatro implementaron intervenciones destinadas a la prevención de la violencia sexual, todas en Estados Unidos y con muestra de estudiantes. Dos de los estudios desarrollaron un programa de prevención de violación como curso dentro de un semestre académico, uno consideró un programa de educación en victimología y un último estudio realizó una intervención que estuvo enfocada en la lectura de un libro, donde se relata la historia de una adolescente que fue víctima de violación (Baldwin-White et al., 2016; Edwards et al., 2019; Fox \& Cook, 20I I; Malo-Juvera, 20 I2; Zinzow et al., 20 I8). Además, otros tres estudios evaluaron capacitaciones específicas en ámbitos de prevención y conocimientos acerca de las agresiones sexuales, implementadas por las organizaciones a las que pertenecían los participantes. Una de las capacitaciones se orientó a oficiales de policía, otra a estudiantes secundarios y la última a estudiantes universitarios del ámbito de la justicia (Campbell et al., 2019; Edwards et al., 2019; Kim \& Santiago, 2019).

\section{Resultados de la relación de los mitos de violación con otras variables}

Los artículos analizados indican que el sexo es un fuerte predictor de la aceptación de los mitos de violación, siendo los hombres los que mayor puntuación obtienen en las distintas medidas aplicadas. Esta diferencia se mantiene en la gran mayoría de los estudios independiente de las características de la muestra (Beshers, 2019; Hayes et al., 20 I6; Manoussaki \& Veitch, 20 I 5; Rominski et al., 2016; Schulze \& Koon-Magnin, 2017; Stephens et al., 2016; Worthen, 2017).

Dentro de las variables ideológicas el sexismo en sus diferentes expresiones, y en particular el sexismo hostil, parece ser el predictor más importante de los mitos de violación. Estos resultados se reportan sin variaciones relevantes a lo largo de los once años de investigación (Custers \& McNallie, 2016; Hill \& Marshall, 2018; Manoussaki \& Hayne, 2019; Poerwandari et al., 2019; Rollero \& Tartaglia, 2018; Stoll et al., 2018). Otras de las variables ideológicas que evidencian tener relación positiva con los mitos de violación son: religiosidad, autoritarismo de derecha, creencia de un mundo justo y dominancia social (Canto et al., 20l4; Ensz \& Jankowski, 20l7; Hammond et al., 20II; Matthews et al., 2018; Navarro \& Tewksbury, 2017a, 2017b; Romero-Sánchez et al., 2017).

Se plantea que el sexo, el género y la religiosidad son variables de alto poder predictivo de los mitos de violación (Barnett et al., 2016; Reling et al., 2017). Sin embargo, Navarro y Tewksbury (2016) sostienen que el nivel de la creencia religiosa no es el factor que predice directamente la aceptación del mito de la violación, sino es su interacción con el tipo de creencia. Por ejemplo, los católicos altamente religiosos poseen bajos niveles de mitos de violación, pero los católicos que son menos religiosos se adhieren más a estos mitos. También argumentan que existe un efecto de interacción importante entre sexo y religiosidad, pues los hombres católicos y protestantes presentan mayor adhesión a los mitos de violación que las mujeres, mientras que las personas que se consideran ateas presentan menores niveles de adhesión, sin diferencias entre hombres y mujeres (Navarro \& Tewksbury, 2016).

Las variables asociadas a la sexualidad muestran que las mujeres con mayor aceptación de mitos de violación son menos asertivas en el rechazo sexual, poseen pocas actitudes positivas en el consentimiento sexual y muestran conductas sexuales autorreprimidas y bajo reconocimiento de los actos 
de violación (Kilimnik \& Humphreys, 2018; Newins et al., 2018; Rinehart et al., 2018; Silver \& Hovick, 2018). Adicionalmente, mayor nivel de aceptación de mitos de violación disminuye la probabilidad de denunciar actos de violación, tanto en víctimas hombres como víctimas mujeres (Hahn et al., 2018). El doble estándar sexual, que se caracteriza por asumir que los hombres deben tener un rol sexualmente dominante, mientras que las mujeres deben asumir un rol pasivo, inexperto y sexualmente inhibido; junto a las creencias sexuales disfuncionales, las que se asocian a la idea de que el deseo en la mujer es pecado; y la preocupación por el prestigio de la mujer, son variables que se ligan positivamente con la aceptación de mitos de violación (Arañez, 2019; Barnett et al., 2017; Lee et al., 2009; Walfield, 2018).

Gran parte de los estudios reportaron relaciones significativas de los mitos de violación con variables asociadas a la violencia interpersonal y de género. Las personas que tienen mayor adhesión a los mitos de violación muestran puntajes elevados en escalas que aceptan la violencia de género, sexual y proclividad de cometer violación (Chapleau \& Oswald, 2010; Finchilescu \& Dugard, 2018; Saucier et al., 2015; Vance et al., 2015). Además, las personas expuestas a experiencias directas de victimización sexual, así como exposición indirecta (haber tenido contacto con alguien que haya sufrido este tipo de violencia), tienen menor aceptación de mitos de violación (RojasAshe et al., 2019; Vonderhaar et al., 2014).

Los mitos de violación medidos en muestras de agentes policiales muestran que los hombres y mujeres policías tienden a respaldar en mayor medida mitos asociados a la falta de credibilidad de la víctima. Sin embargo, no se observan diferencias significativas con relación a los otros mitos de violación comparados con otras muestras (Sleath \& Bull, 2015). Por otro lado, cuando se introduce el consumo de alcohol por parte de la víctima, los policías disminuyen las acciones que agilizan la investigación y la detención del agresor en escenarios simulados (Venema, 2016). La actitud de los policías hacia las mujeres y las víctimas, la percepción de responsabilidad de la víctima en las agresiones sexuales y el sexismo se enlazan positivamente con los mitos de violación (Lee et al., 20II; Smith et al., 20I5). Murphy y Hine (2018) plantean que los constructos actitudinales explican una proporción mucho mayor de la varianza en los mitos de violación al compararlos con variables sociodemográficas.

Desde el punto de vista de las víctimas, las personas con alta aceptación de mitos de violación tienden a no reconocerse como víctimas, excusando el comportamiento sexual agresivo de los hombres
(Newins et al., 2018). Por otro lado, el reconocerse como víctima de agresión sexual tiene efectos diferenciales dependiendo del nivel de aceptación de los mitos de violación de las víctimas. Las víctimas de violación con alto RMA, que reconocen la agresión sexual, tienen mayor sintomatología depresiva y mayor consumo de alcohol; las víctimas que no reconocen haber sido agredidas sexualmente poseen menos síntomas depresivos y menor consumo de alcohol. También, las personas con baja aceptación de mitos de violación, y que no reconocen la violación, informaron peores resultados en síntomas depresivos y consumo de alcohol, que las víctimas de violación que reconocen haber sido violentadas (Wilson et al., 2017).

En cuanto al consumo de alcohol los hombres con alto consumo muestran mayor respaldo de los mitos de violación. Además, un $28 \%$ de los hombres reconoce haber dado alcohol a mujeres para acceder sexualmente a ellas, no admitiendo este acto como violación. Según algunos autores, el alcohol no es simplemente una herramienta utilizada para la violación o el abuso, sino que forma parte de la cultura de la violación (Hayes et al., 2016; RomeroSánchez et al., 2017).

Los estudios que vinculan las características de personalidad con la aceptación del mito de violación muestran que los sujetos con personalidad psicopática, en particular los rasgos de insensibilidad y manipulación, tienen mayor aceptación de los mitos de violación que responsabilizan a la víctima (Debowska, 20I5; Mouilso \& Calhoun, 20I3). El mito que plantea que la violación no ocurrió y que es una exageración de la víctima se asocia al comportamiento impulsivo y antisocial, lo que posiblemente sugiere que este mito está relacionado con una mayor tendencia a excusar el comportamiento agresivo (Willis et al., 2017; Mouilso \& Calhoun, 2013). Además, los mitos de violación y las cogniciones de una sexualidad agresiva revelan asociaciones con rasgos como el antagonismo, la desinhibición y la afectividad negativa, en su polaridad patológica (Kasowski \& Anderson, 2019).

Los resultados de los estudios cuasiexperimentales indican que la aceptación de mitos de violación ejerce un efecto de sesgo en el procesamiento de cualquier información relevante acerca de la agresión sexual, dirigiendo la memoria, atención y percepción hacia los estímulos contextuales, que justifican la responsabilidad de la víctima de violación. Por ejemplo, poner mayor atención a la cantidad de alcohol consumido y omitir información que da cuenta que la víctima rechazó el contacto de alguna forma (Dawtry et al., 2018; Duff \& Tostevin, 2015; Eyssel \& Bohner, 20 I0; Süssenbach et al., 2012; Süssenbach et al., 2015). Estos sesgos también afectan la creencia acerca de la 
expresión emocional esperada en las víctimas. Las personas con alta aceptación de mitos de violación ven como más creíble a la víctima que expresa tristeza, considerando menos creíbles a las víctimas que expresan rabia. Otro estereotipo que genera sesgo en los procesos atribucionales es la creencia de que la víctima sería la responsable de la violación, si esta acepta bebidas alcohólicas ofrecidas por el agresor (Bohner \& Schapansky, 2018; Romero-Sánchez et al., 2017). La exposición a contenidos metafóricos, donde se representa a los hombres como depredadores y a las mujeres como presas, aumentaría la aceptación del mito de la violación (Bock \& Burkley, 2019).

También se evidencia en estos estudios de corte experimental que la participación en programas de intervención destinados a la prevención de la violencia sexual, mediante la educación en temas relativos a la victimización, violencia sexual, prevención deconductas de riesgo de agresión sexual asociadas principalmente al consumo de alcohol y drogas, y la promoción de acciones destinadas al cuidado de los pares en caso de riesgo de victimización, disminuyen significativamente los niveles de aceptación de mitos de violación, efecto que se mantiene relativamente estable en el tiempo (Baldwin-White et al., 2016; Campbell et al., 2019; Edwards et al., 2019; Fox \& Cook, 20I I; MaloJuvera, 2012; Vance et al., 20I5; Zinzow et al., 20I8). Dentro de estos resultados las variables asociadas a las intervenciones en prevención de agresión sexual abarcaron temas relativos a consentimiento sexual, características en la clasificación jurídica de los delitos sexuales, consecuencias del consumo excesivo de alcohol y otras drogas; victimización, trauma y herramientas conductuales para intervenir en situaciones potencialmente riesgosas para terceros en el caso de estar observando una situación de acoso - impedimento para consentir o resistir un ataque sexual (Campbell et al., 2019; Baldwin-White et al., 2016; Edwards et al., 2019; Zinzow et al., 2018).

\section{Discusión}

El propósito del presente trabajo fue revisar y sintetizar los estudios empíricos que han utilizado la teoría de mitos de violación entre los años 2009 y 2019. Siguiendo las recomendaciones de la declaración Prisma se realizó una búsqueda en seis bases de datos (WoS, Scopus, ProQuest, PsycINFO, EBSCOhost y JSTOR), selección que agrupó un total de 96 artículos publicados. La mayoría de los estudios fueron norteamericanos y europeos, ejecutados con estudiantes universitarios y con diseños correlacionales.

Entre los resultados más relevantes se evidencia que los hombres en general aceptan en mayor medida los mitos de violación que las mujeres y que esta aceptación también se asocia a actitudes negativas hacia las mujeres y mayor adherencia a creencias ideológicas como: sexismo, creencia de un mundo justo, dominancia social, autoritarismo y religiosidad. Por tanto, podemos inferir que las ideologías conservadoras mantienen y justifican los sesgos respecto a la violencia sexual, reproduciendo creencias sobre la responsabilidad en la exposición a la ocurrencia del delito, así como la posibilidad de haberlo evitado (Romero-Sánchez, 2012). Además, las personas que han estado en contacto con víctimas de agresión sexual, en forma directa o indirecta, que no comparten los estereotipos tradicionales de género o que tienen formación en temas de victimología, tienden a tener baja aceptación de los mitos de violación y menor respaldo a los victimarios en comparación al resto de las personas.

También se observa que algunas víctimas de violación son renuentes a denunciar. Esto está asociado a que no se reconocen como víctimas, dado que asumen la culpa de la agresión o por miedo a ser cuestionada su credibilidad. La posibilidad de perder prestigio social, siendo sindicada como el tipo de mujer que es susceptible de ser violada, ya sea por la exposición o por su actuar, parece ser un factor relacionado con el no reconocimiento de la agresión (Burt, 1980). Las mujeres con alta aceptación de mitos y que no se reconocen como víctimas, tienen menor sintomatología depresiva, lo que podría ser explicado por la normalización de la violencia sexual, asumiendo estos actos como parte de las dinámicas de las relaciones sexuales. Las mujeres que se reconocen como víctimas y tienen baja aceptación de mitos de violación tienen menor probabilidad de presentar problemas de salud mental (Wilson et al., 2017). Esto se puede explicar dado que depositan la culpa en el agresor, disminuyendo las emociones y pensamiento negativo respecto a su participación y responsabilidad en la violación.

Los estudios que emplearon intervenciones destinadas a la prevención de las agresiones sexuales plantean que estos programas deben poseer un enfoque integrador (Kim \& Santiago, 2019; Zinzow et al., 2018), que sean capaces de aproximarse al fenómeno desde diversas áreas del conocimiento, permitan complementar los contenidos mínimos necesarios para abordar las agresiones sexuales y 
ayuden a disminuir las creencias erróneas con respecto a la violación. Dentro de los saberes necesarios para implementar un programa de prevención de las agresiones sexuales para su aplicación en población general, es necesario incluir conocimientos en: (a) victimología y legislación de los delitos sexuales; (b) consecuencias del consumo excesivo de alcohol y otras drogas, conductas de riesgo y salud mental; (c) sexualidad, diversidad sexual y género y (d) conocer los sistemas de creencias de los participantes con respecto a la violación (Baldwin-White et al., 2016; Hill \& Marshall, 2018; Malo-Juvera, 20I2; Zinzow et al., 2018). Aparentemente no es suficiente intervenir en el problema de la violencia sexual solo desde la educación sexual, dado que el sistema que mantiene estos mitos está relacionado con estructuras ideológicas y creencias falsas fuertemente arraigadas, que justifican el actuar del agresor y la culpabilidad de la víctima (Canto et al., 20l4; Lutz-Zois et al., 20I5; Kim \& Santiago, 2019). El desafío para futuras investigaciones debe concentrarse en evaluar la eficacia de programas de prevención de la violencia sexual, que integren dentro de sus dimensiones los cuatro puntos antes descritos. Dada la escasa evidencia de estudios aplicados que incorporen programas de prevención asociados a los mitos de violación y considerando el alto porcentaje de violencia sexual reportados en Latinoamérica y el Caribe (García-Moreno et al., 20I3), resulta relevante y necesario promover líneas de investigación que puedan entregar conocimientos pertinentes adaptados a la población latinoamericana.

Teniendo en cuenta los hallazgos de los estudios realizados con personas víctimas de violación es menester que futuras investigaciones se dirijan a identificar las conductas de autocuidado que mejoren la asertividad en el rechazo y el consentimiento sexual, para que se incorporen en los programas con destino a disminuir la revictimización sexual y las consecuencias socioemocionales de la violación. Es necesario considerar estas variables en las intervenciones con víctimas, pues la evidencia plantea que la falta o baja expresión de estas variables se asocia a mayor riesgo de revictimización, a menor probabilidad de denunciar una agresión y a baja capacidad para reconocer la victimización sexual (Kilimnik \& Humphreys, 2018; Newins et al., 2018; Silver \& Hovick, 2018). Sin duda, el desarrollo de estudios en esta línea es vital para recabar mayor evidencia empírica que permita el diseño de programas e intervenciones que favorezcan el reconocimiento y denuncia por parte de las víctimas, así como reducir la revictimización sexual.

La evidencia presentada trae algunas limitaciones. La mayoría de los participantes en los estudios son estadounidenses y europeos, principalmente estudiantes universitarios, en un alto porcentaje pertenecientes a países occidentales, con alto nivel educativo, industrializados, ricos y democráticos (western, educated, industrialized, rich and democratic) (Henrich et al., 20l0). Esta es una limitación frecuente de las investigaciones psicosociales, por lo que es urgente indagar la aceptación de mitos de violación en otros contextos socioculturales (Henrich et al., 20l0). Una segunda limitación es que los mitos de violación son una variable sensible a la deseabilidad social. No obstante, en pocos estudios se valora la influencia de esta variable (Saucier et al., 20I5; Hockett et al., 2009; Lutz-Zois et al., 2015; Walfield, 2018).

La mayoría de los estudios abordan el fenómeno de los mitos de violación en mujeres existiendo escasa evidencia que pueda otorgar información con respecto al fenómeno de los hombres víctimas de violencia sexual. Siendo parte de estos mitos la creencia de que los hombres no pueden sufrir violencia sexual por parte de mujeres u otros hombres (Davies et al., 2012; Klement et al., 2018; Reling et al., 2017; Walfield, 2018). La mayoría de los instrumentos de aceptación de mitos de violación traen implícito que el perpetrador es un hombre. Si bien el concepto de violación desde una perspectiva jurídica atribuye que la violación es un acto exclusivamente masculino (Carrasco, 2007), no podemos negar que la agresión sexual no se circunscribe a mujeres víctimas y hombres perpetradores (Walfield, 2018).

Las investigaciones abarcan una gran cantidad de variables que se relacionan con los mitos de violación. Sin embargo, muy pocas tratan de entregar relaciones causales entre las variables, por lo que es necesario desarrollar investigaciones de corte experimental que logren contrastar modelos explicativos acerca de los procesos atribucionales en casos de violencia sexual. Esto es un desafío para nuevas líneas de investigación.

La mayor cantidad de estudios utiliza medidas de RMA creadas y validadas en Estados Unidos y en población universitaria, no existiendo aún evidencia acerca de la equivalencia/invarianza de media en otros contextos culturales o grupos etarios diversos (Barn \& Powers, 2018).

La sistematización de estudios empíricos de los mitos de violación permite formar una visión general de las líneas de investigación que se han desarrollado en torno a este constructo teórico, probando la necesidad de contribuir con investigación que ayude a explicar desde la realidad latinoamericana la construcción de estas creencias sesgadas acerca de la violencia sexual. Sin duda, conocer el estado del arte de los mitos de violación facilitará orientar las 
intervenciones en las políticas públicas para prevenir y disminuir la violencia sexual, principalmente hacia las mujeres, mediante programas de educación dirigidos a la población general. Adicionalmente, permitiría mejorar las prácticas del sistema de justicia y de sus intervinientes, para disminuir los sesgos que podrían explicar las bajas tasas de denuncias y sanciones en caso de delitos sexuales (Montes, 2012). Desde el trabajo directo con las personas que han sido víctimas de agresión, la visibilización y abordaje de los mitos internalizados en las víctimas permitirán disminuir la atribución de la culpa, favoreciendo la reconstrucción de la vivencia sin la carga emocional que implica asumir la responsabilidad del acto de violación.

\section{Conflicto de intereses}

Los autores hacen constar que no existe ningún conflicto de intereses reales o potenciales derivados de la elaboración del presente artículo.

\section{Referencias}

Arañez, S. D. (2019). She's just a prostitute: the effects of labels on counselor attitudes, empathy, and rape myth acceptance. Professional Counselor, 9(4), 396-4I5. https://doi.org//0.1524I/ sdal.9.4.396

Aronowitz, T., Lambert, C. A., \& Davidoff, S. (20I2). The role of rape myth acceptance in the social norms regarding sexual behavior among college students. Journal of Community Health Nursing, 29(3), 173-182. https://doi.or g/l0.1080/07370016.2012.697852

Baldwin-White, A., Thompson, M. S., \& Gray, A. (20I6). Pre-and postintervention factor analysis of the Illinois rape myth acceptance scale. Journal of Aggression, Maltreatment \& Trauma, 25(6), 636-65I. https://doi.org/10.10 80/1092677I.2015.1107173

Barn, R., \& Powers, R. A. (20I8). Rape myth acceptance in contemporary times: a comparative study of university students in India and the United Kingdom. Journal of Interpersonal Violence, 36(7-8), 3514-3535. https://doi. org/I0.II77/08862605/8775750

Barnett, M. D., Hale, T. M., \& Sligar, K. B. (20I7). Masculinity, femininity, sexual dysfunctional beliefs, and rape myth acceptance among heterosexual college men and women. Sexuality \& Culture, 2I(3), 74I-753. https://doi.org/ I0.1007/s I 2 I 19-0 I7-9420-3

Barnett, M. D., Sligar, K. B., \& Wang, C. D. C. (20I6). Religious affiliation, religiosity, gender, and rape myth acceptance: feminist theory and rape culture. Journal of Interpersonal Violence, 33(8), 1219-1235. https://doi. org/I0. I I77/08862605I6665II0

Baugher, S. N., Elhai, J. D., Monroe, J. R., \& Gray, M. J. (20I0). Rape myth acceptance, sexual trauma history, and posttraumatic stress disorder. Journal of Interpersonal Violence, 25(II), 2036-2053. https://doi. org/l0.I I 77/0886260509354506

Beshers, S., \& DiVita, M. (2019). Changes in rape myth acceptance among undergraduates: 2010 to 2017. Journal of Interpersonal Violence. https:// doi.org//0.1 I77/08862605/9867/53

Bhogal, M. S., \& Corbett, S. (2016). The influence of aggressiveness on rape-myth acceptance among university students. Psychology and Law, 23(5), 709-7I5. https://doi.org//0.1080/ | 32 | $8719.2016 .1 \mid 4293$ |

Bock, J., \& Burkley, M. (2019). On the prowl: examining the impact of men-as-predators and womenas-prey metaphors on attitudes that perpetuate sexual violence. Sex Roles, 80(56), 262-276. https://doi.org//0.1007/s I I I99018-0929-1

Bohner, G. (2007). Acceptance of modern myths about sexual aggression. PsycTESTS Dataset. https://doi.org//0.1037/t02749-000

Bohner, G., \& Schapansky, E. (20I8). Law students' judgments of a rape victim's statement: the role of displays of emotion and acceptance of sexual aggression myths. International Journal of Conflict and Violence, 12, a635. https://doi. org/I0.4I I9/unibi/ijcv.635

Burgess, G. H. (2007). Assessment of rape supportive attitudes and beliefs in college men. Journal of Interpersonal Violence, 22, 973-993. https://doi. org/l0.1 I 77/0886260507302993

Burt, M. R. (1980). Cultural myths and supports for rape. Journal of Personality and Social Psychology, 38, 2 17-230.

Campbell, B. A., Lapsey, D. S., \& Wells, W. (2019). An evaluation of Kentucky's sexual assault investigator training: results from a randomized three-group experiment. Journal of Experimental Criminology, I-23. https://doi. org/ I0.1007/s I I292-019-0939I-0

Canan, S. N., Jozkowski, K. N., \& Crawford, B. L. (20I6). Sexual assault supportive attitudes: rape myth acceptance and token resistance in greek and non-greek college students from two university samples in the United States. Journal of Interpersonal Violence, 33(22), 3502-3530. https://doi. org/l0.1 I77/08862605I6636064

Canto, J. M., Perles, F., \& Martín, J. S. (2014). The role of right-wing authoritarianism, sexism and culture of honour in rape myths acceptance. Revista de Psicología Social, 29(2), 
296-3/8. https://doi.org/10.1080/02134748.2 014.918822

Carrasco, E. (2007). El problema del sujeto activo del delito de violación y sus posibles vacíos legales. Lus et Praxis, 13(2), I37-155. https://dx.doi. org/l 0.4067/S07/8-00I22007000200007

Carroll, M. H., Rosenstein, J. E., Foubert, J. D., Clark, M. D., \& Korenman, L. M. (20I6). Rape myth acceptance: a comparison of military service academy and civilian fraternity and sorority students. Military Psychology, 28(5), 306-317. https://doi.org/I0.1037/mil0000II 3

Chapleau, K. M., \& Oswald, D. L. (2010). Power, sex, and rape myth acceptance: testing two models of rape proclivity. Journal of Sex Research, 47(I), 66-78. https://doi. org/l 0.1080/00224490902954323

Comisión Interamericana de Derechos Humanos. (20II). Acceso a la justicia para mujeres víctimas de violencia sexual: la educación y la salud.Organización delosEstadosAmericanos. https://www.oas.org/es/cidh/mujeres/docs/ pdf/VIOLENCIASEXUALEducySalud.pdf

Contreras, J. M., Both, S., Guedes, A., \& Dartnall, E. (2016). Violencia sexual en Latinoamérica y el Caribe: análisis de datos secundarios. Iniciativa de investigación sobre la violencia sexual. Consorcio Latinoamericano contra el Aborto Inseguro. http://clacaidigital.info/handle/I 23456789/980

Custers, K., \& McNallie, J. (2016). The relationship between television sports exposure and rape myth acceptance: the mediating role of sexism and sexual objectification of women. Violence Against Women, 23(7), 813-829. https://doi. org/I0.1 I77//07780I2I665I340

Da Silva, T., Woodhams, J., \& Harkins, L. (2018). An adventure that went wrong: reasons given by convicted perpetrators of multiple perpetrator sexual offending for their involvement in the offense. Archives of Sexual Behavior, 47, 443-456. https://doi. org/10.1007/s 10508-017-101 I-8

Davies, M., Gilston, J., \& Rogers, P. (20I2). Examining the relationship between male rape myth acceptance, female rape myth acceptance, victim blame, homophobia, gender roles, and ambivalent sexism. Journal of Interpersonal Violence, 27(14), 2807-2823. https://doi. org/| 0.1 I 77/08862605 I 243828 I

Dawtry, R. J., Cozzolino, P. J., \& Callan, M. J. (2018). I blame therefore it was: rape myth acceptance, victim blaming, and memory reconstruction. Personality and Social Psychology Bulletin, 45(8), I269-I282. https:// doi.org/|0.1 I77/0146167218818475
Debowska, A., Boduszek, D., Dhingra, K., \& DeLisi, M. (2016). The effect of male incarceration on rape myth acceptance: application of propensity score matching technique. Deviant Behavior, 37(6), 634-643. https://doi.org/10.10 80/01639625.2015.1060805

Debowska, A., Boduszek, D., Dhingra, K., Kola, S., \& Meller-Prunska, A. (2015). The role of psychopathy and exposure to violence in rape myth acceptance. Journal of interpersonal violence, 30(15), 275I-2770. https://doi. org/ I0.I I77/08862605|4553635

Dellinger, A. (2010). True colors: police officers and rape myth acceptance. Feminist Criminology, 5(4), 315-334. https://doi. org/I0.1 I77/I557085II0384I08

Duff, S., \& Tostevin, A. (20I5). Effects of gender, rape myth acceptance, and perpetrator occupation on perceptions of rape. Journal of Criminal Psychology, 5(4), 249-26I. https://doi. org/I0.1 I08/JCP-I2-2014-0019

Dworkin, E. R., Sessarego, S. N., Pittenger, S. L., Edwards, K. M., \& Banyard, V. L. (2017). Rape myth acceptance in sexually assaulted adolescents' school contexts: associations with depressed mood and alcohol use. American Journal of Community Psychology, 60(3-4), 516-526. https://doi. org/10.1002/ajcp. 12173

Edwards, K. M., Banyard, V. L., Sessarego, S. N., Waterman, E. A., Mitchell, K. J., \& Chang, H. (2019). Evaluation of a bystander-focused interpersonal violence prevention program with high school students. Prevention Science, 20(4), 488-498. https://doi.org//0.1007/ sIII2I-019-01000-W

Egan, R., \& Wilson, J. C. (20I2). Rape victims' attitudes to rape myth acceptance. Psychiatry, Psychology and Law, 19(3), 345-357. https://doi.org/10.10 $80 / 13218719.2011 .585128$

Elliott, D. M., Mok, D. S., \& Briere, J. (2004). Adult sexual assault: prevalence, symptomatology, and sex differences in the general population. Journal ofTraumatic Stress, I 7(3), 203-2I I. https://doi. org/ I 0.1023/b:jots.0000029263. I I I 04.23

Emmers-Sommer, T. M. (2017). College student perceptions of hypothetical rape disclosures: do relational and demographic variables pose a risk on disclosure believability? Sexuality \& Culture, $2 I(3)$, 664-679. https://doi. org/|0.1007/s I 2 I |9-0 I7-94| I-4

Ensz, S., \& Jankowski, P. J. (20I7). Religiousness and rape myth acceptance. Journal of Interpersonal Violence, 35(7-8), I67|-1693. https://doi. org/|0.1 I77/08862605 I769828 | 
Eyssel, F., \& Bohner, G. (20I0). Schema effects of rape myth acceptance on judgments of guilt and blame in rape cases: the role of perceived entitlement to judge. Journal of Interpersonal Violence, 26(8), 1579-1605. https://doi. org//0.1 I77/08862605/0370593

Fattah, E. (20I4). Victimología: pasado, presente y futuro. Revista Electrónica de Ciencia Penal y Criminología, 33(I), I-33. https:// ns I.justucuman.gov.ar/archivos/ entradas/208//488887229.pdf

Finchilescu, G., \& Dugard, J. (20|8). Experiences of gender-based violence at a South African university: prevalence and effect on rape myth acceptance. Journal of Interpersonal Violence, 36(5-6), NP2749-NP2772. https:// doi.org//0.II 77//08862605/8769352

Fox, J., \& Potocki, B. (2015). Lifetime video game consumption, interpersonal aggression, hostile sexism, and rape myth acceptance: a cultivation perspective. Journal of Interpersonal Violence, 3I(10), 1912-1931. https://doi. org/I0.II I7//08862605/5570747

Fox, K. A., \& Cook, C. L. (20II). Is knowledge power? The effects of a victimology course on victim blaming. Journal of Interpersonal Violence, 26(I7), 3407-3427. https://doi. org// 0.1 I 777/08862605 I I 403752

García-Moreno, C., Pallitto, C., Devries, K., Stöckl, H., Watts, C., \& Abrahams, N. (20I3). Global and regional estimates of violence against women: prevalence and health e ects of intimate partner violence and non-partner sexual violence. World Health Organization. http://apps.who.int/iris/ bitstream//0665/85239/I/978924I564625_ eng.pdf

Gerger, H., Kley, H., Bohner, G., \& Siebler, F. (2007). The acceptance of modern myths about sexual aggression scale: development and validation in German and English. Aggressive Behavior, 33(5), 422-440. https://doi. org/I0.1002/ab.20195

Grubb, A., \& Turner, E. (20I2). Attribution of blame in rape cases: a review of the impact of rape myth acceptance, gender role conformity and substance use on victim blaming. Aggression and Violent Behavior, I7(5), 443-452. https:// doi.org/I0.10I6/j.avb.20I2.06.002

Hahn, C. K., Hahn, A. M., Gaster, S., \& Quevillon, R. (20I8). Predictors of college students' likelihood to report hypothetical rape: rape myth acceptance, perceived barriers to reporting, and self-efficacy. Ethics \& Behavior, I-I8. https://doi.org//0.1080/10508422.2018. |552519
Hammond, E. M., Berry, M. A., \& Rodríguez, D. N. (20II). The influence of rape myth acceptance, sexual attitudes, and belief in a just world on attributions of responsibility in a date rape scenario. Legal and Criminological Psychology, 16(2), 242-252. https://doi. org/I0.1348//355325I0x499887

Hayes, R. M., Abbott, R. L., \& Cook, S. (2016). It's her fault. Violence Against Women, 22(13), I540-1555. https://doi. org//0.1I77/I07780I2I6630I47

Hayes-Smith, R. M., \& Levett, L. M. (20I0). Student perceptions of sexual assault resources and prevalence of rape myth attitudes. Feminist Criminology, 5(4), 335-354. https://doi. org/ I0.1 I77/I557085 I I038758 |

Henrich, J., Heine, S. J., \& Norenzayan, A. (2010). Most people are not WEIRD. Nature, 466(7302), 29. https://doi.org/I 0.1038/466029a

Henry, D. S., Merrell, L. K., Blackstone, S. R., CollazoVargas, E., Mohl, C., Tolerico, M., Singley, L., \& Moody, S. (2019). Does perpetrator occupation affect classification of sexual assault? Journal of Interpersonal Violence, 36(19-20), 10255-10275. https://doi. org/I0.1 I 77/08862605/987333।

Hill, S., \& Marshall, T. C. (20I8). Beliefs about sexual assault in India and Britain are explained by attitudes toward women and hostile sexism. Sex Roles, 79(7-8), 42I-430. https://doi. org/| 0.1007/s I I I 99-017-0880-6

Hine, B., \& Murphy, A. (2018). The influence of 'High' vs. 'Low' rape myth acceptance on police officers' judgements of victim and perpetrator responsibility, and rape authenticity. Journal of Criminal Justice, 60, 100-107. https://doi. org/10.1016/j.jcrimjus.2018.08.00I

Hockett, J. M., Saucier, D. A., \& Badke, C. (2016). Rape myths, rape scripts, and common rape experiences of college women: differences in perceptions of women who have been raped. Violence Against Women, 22(3), 307-323. https://doi.org//0.1 I77/107780 I 2/5599844

Hockett, J. M., Saucier, D. A., Hoffman, B. H., Smith, S. J., \& Craig, A. W. (2009). Oppression through acceptance? Violence Against Women, 15(8), 877-897. https://doi. org/I 0.I I 77// 07780I 209335489

Instituto Nacional de Medicina Legal y Ciencias Forenses [INMLCF]. (2019). Forensis 2018: datos para la vida. Grupo Centro de Referencia Nacional sobre Violencia, Bogotá, D.C. (la ed.). https://www.medicinalegal.gov.co/ documents/20 I43/386932/Forensis+20I8. pdf 
Javaid, A. (2015). Male rape myths: understanding and explaining social attitudes surrounding male rape. Masculinities \& Social Change, 4(3), 270-297. https://doi. org//0.1 I 77// 07780 I2/5599844

Kasowski, A. E., \&Anderson,J.L.(2019). The association between sexually aggressive cognitions and pathological personality traits in men. Violence Against Women, 26(12-13), 1636-1655. https:// doi.org//0.1 I77/1077801219873436

Kaukinen, C. \& DeMaris, A. (2005). Age at first sexual assault and current substance use and depression. Journal of Interpersonal Violence, 20(10), 1244-1270. https://doi. org/l 0.1 I 77/ 0886260505277732

Kilimnik, C. D., \& Humphreys, T. P. (2018). Understanding sexual consent and nonconsensual sexual experiences in undergraduate women: the role of identification and rape myth acceptance. The Canadian Journal of Human Sexuality, 27(3), 195-206. https://doi.org//0.3/38/cjhs.20170028

Kim, B., \& Santiago, H. (2019). Rape myth acceptance among prospective criminal justice professionals. Women \& Criminal Justice. https://doi.org//0.1080/08974454.2019.1664 969

King, L. L., \& Hanrahan, K. J. (20I5). University student beliefs about sexual violence in prison: rape myth acceptance, punitiveness and empathy. Journal of Sexual Aggression, 2I(2), 179-193. https://doi.org//0.1080//3552600.2 $0|3.82085|$

King, L. L., \& Roberts, J. J. (20I I). Traditional gender role and rape myth acceptance: from the countryside to the big city. Women \& Criminal Justice, 2 I (I), I-20. https://doi.org/I0.1080/08 974454.20II.536045

Klement, K. R., Sagarin, B. J., \& Skowronski, J. J. (20/8). Accusers lie and other myths: rape myth acceptance predicts judgments made about accusers and accused perpetrators in a rape case. Sex Roles, $8 /(I-2)$, 16-33. https:// doi.org/| 0.1007/s I | |99-0| |8-0950-4

Lee, J., Kim, J., \& Lim, H. (2009). Rape myth acceptance among Korean college students: the roles of gender, attitudes toward women, and sexual double standard. Journal of Interpersonal Violence, 25(7), 1200-1223. https://doi. org/ 10.1 I 77/0886260509340536

Lee, J., Lee, C., \& Lee, W. (20II). Attitudes toward women, rape myths, and rape perceptions among male police officers in South Korea. Psychology of Women Quarterly, 36(3), 365-376. https://doi.org/|0.1 |77/036|6843||427538
LeMaire, K. L., Oswald, D. L., \& Russell, B. L. (2016). Labeling sexual victimization experiences: the role of sexism, rape myth acceptance, and tolerance for sexual harassment. Violence and Victims, 3I/(2), 332-346. https://doi. org/| 0.189|/0886-6708.VV-D-|3-00|48

Liberati, A., Altman, D. G., Tetzlaff, J., Mulrow, C., Gotzsche, P. C., loannidis, J. P., Clarke, M., \& Moher, D. (2009). The PRISMA statement for reporting systematic reviews and metaanalyses of studies that evaluate health care interventions: explanation and elaboration. Journal of Clinical Epidemiology, 62(10), el-e34. https://doi.org//0.1016/j.jclinepi.2009.06.006

Lutz-Zois, C. J., Moler, K. A., \& Brown, M. J. (20I5). Mechanisms for the relationship between traditional masculine ideologies and rape myth acceptance among college men. Journal of Aggression, Maltreatment \& Trauma, 24(I), 84-I0I. https://doi.org/I0.1080/I092677I.20 |5.9963| |

Malo-Juvera, V. (2012). The effect of young adult literature on adolescents rape myth acceptance. [Tesis doctoral]. Florida International University. https://doi. org/I0.25 I48/etd.FII204III 5

Manoussaki, K., \& Hayne, A. (2019). Authoritarianism, social dominance, religiosity and ambivalent sexism as predictors of rape myth acceptance. International Journal of Gender and Women's Studies, 7(I), 79-84. https://doi. org/I0.15640/ijgws.v7n la I0

Manoussaki, K., \& Veitch, F. (20I5). Ambivalent sexism, right wing authoritarianism and rape myth acceptance in Scotland. International Journal of Gender \& Women's Studies, 3(1). https://doi.org/I0.15640/ijgws.v3n la9

Matthews, J., Avery, L., \& Nashandi, J. (20I8). Southern African social work students' acceptance of rape myths: results from an exploratory study. Social Sciences, 7, 152. https://doi. org/ I0.20944/preprints20 I808.0 I I5.v I

Maxwell, L., \& Scott, G. (20I4). A review of the role of radical feminist theories in the understanding of rape myth acceptance. Journal of Sexual Aggression, 20(I), 40-54. https://doi.org//0.10 $80 /|3552600.20| 3.773384$

McMahon, S. (2010). Rape myth beliefs and bystander attitudes among incoming college students. Journal of American College Health, 59(I), 3-II. https://doi.org/I0.1080/0744848I.2010.48 3715

McMahon, S., \& Farmer, G. L. (20II). An updated measure for assessing subtle rape myths. Social Work Research, 35(2), 7I- 8I. https:// doi.org/I0.1093/swr/35.2.7I 
Melanson, P. S. K. (1998). Belief in male rape myths: a test of two competing theories. [Tesis doctoral]. http://www.collectionscanada. gc.ca/obj/s4/f2/dsk2/tape I5/PQDD_0003/ NQ31935.pdf

Ministerio Público de Chile. (2019). Boletín estadístico III trimestre (enero-septiembre). http://www. fiscaliadechile.cl/Fiscalia/estadisticas/index.do

Miralles, R. (2020). Cultura de la violación: una cuestión política. Libre Pensamiento, 102, 82-87. https:// dialnet.unirioja.es/ejemplar/546776

Montes, R. V. (20/2). Delincuencia sexual y populismo penal en Colombia. URVIO: Revista Latinoamericana de Estudios de Seguridad, I I, 19-32.

Mouilso, E. R., \& Calhoun, K. S. (20/3). The role of rape myth acceptance and psychopathy in sexual assault perpetration. Journal of Aggression, Maltreatment \& Trauma, 22(2), | 59-| 74. https://doi.org//0.1080/1092677|.2 013.743937

Murphy, A., \& Hine, B. (20l8). Investigating the demographic and attitudinal predictors of rape myth acceptance in U.K. police officers: developing an evidence-base for training and professional development. Psychology, Crime \& Law. https://doi. org/l0.1080/1068316X.2018.1503663

Navarro J. C., \& Tewksbury, R. (2016). Deconstructing the associations of religiosity, Christian denominations, and non-religions to rape myth acceptance among university students. Deviant Behavior. https://doi.org/10.1080/016 39625.2016.1260386

Navarro, J. C., \& Tewksbury, R. (2017a). Mythbusters: examining rape myth acceptance among U.S. university students. Journal of Student Affairs Research and Practice, 54(4), 343-356. https:// doi.org/10.1080/1949659I.2017.1289094

Navarro, J. C., \& Tewksbury, R. (20I7b). National comparisons of rape myth acceptance predictors between nonathletes and athletes from multi-institutional settings. Sexual Abuse, 3I(5), 543-559. https://doi. org/10.1 I77/1079063217732790

Newins, A. R., Wilson, L. C., \& White, S. W. (20I8). Rape myth acceptance and rape acknowledgment: the mediating role of sexual refusal assertiveness. Psychiatry Research, 263, I5-21. https://doi.org/10.1016/j. psychres.2018.02.029

Oh, E., \& Neville, H. (2004). Developmentand validation of the Korean rape myth acceptance scale. The Counseling Psychologist, 32(2), 30I-33I. https:// doi.org/ | 0. I |77/00 I 100000326 I35 |

Organización Panamericana de la Salud. (2003). Informe mundial sobre la violencia y la salud: resumen. Oficina Regional para las Américas de la Organización Mundial de la Salud. https:// www.who.int/violence_injury_prevention/ violence/world_report/en/summary_es.pdf

Payne, D., Lonsway, K., \& Fitzgerald, L. (1999). Rape myth acceptance: exploration of its structure and its measurement using the Illinois rape myth acceptance scale. Journal of Research in Personality, 33, 27-68. https://doi.org//0.1006/ jrpe. 1998.2238

Poerwandari, E. K., Utami, C. P., \& Primasari, I. (2019). Ambivalent sexism and sexual objectification of women as predictors of rape myth acceptance among male college students in Greater Jakarta. Current Psychology, I-I0. https://doi.org/|0.1007/s | 2144-019-00500-w

Powers, R. A., Leili, J., Hagman, B., \& Cohn, A. (20I5). The impact of college education on rape myth acceptance, alcohol expectancies, and bystander attitudes. Deviant Behavior, 36(12), 956-973. https://doi.org//0.1080/01639625.2 014.982747

Read, G. L., Lynch, T., \& Matthews, N. L. (2018). Increased cognitive load during video game play reduces rape myth acceptance and hostile sexism after exposure to sexualized female avatars. Sex Roles, 79( I I- I2), 683-698. https://doi.org// 0.1007/s I I I99-018-0905-9

Reling, T. T., Barton, M. S., Becker, S., \& Valasik, M. A. (20I7). Rape myths and hookup culture: an exploratory study of us college students' perceptions. Sex Roles, 78(7-8), 50I-5I4. https://doi.org//0.1007/s I I 199-017-0813-4

Rinehart, J. K., Yeater, E. A., Treat, T. A., \& Viken, R. J. (20I8). Cognitive processes underlying the self-other perspective in women's judgments of sexual victimization risk. Journal of Social and Personal Relationships, 35(10), I381-1399. https://doi.org/I0.II77/02654075 I77/3365

Rojas-Ashe, E. E., Walker, R. V., Holmes, S. C., \& Johnson, D. M. (20/9). Toward a more complete understanding of bystander willingness to help: what role does critical consciousness play? Sex Roles, 8 I (7-8), 4I 5-427. https://doi.org// 0.1007/s I I 199-019-1007-z

Rollero, T. C., \& Tartaglia, S. (2018). The effect of sexism and rape myths on victim blame. Sexuality \& Culture, 23(I), 209-219. https:// doi.org/I0.1007/s I 2 I 19-018-9549-8

Romero-Sánchez, M. (2012). Percepción social de las agresiones sexuales hacia mujeres: el papel del alcohol y los mitos sobre la violación. [Tesis doctoral]. Universidad de Granada. http://hdl.handle.net//048 I/2 I64 I

Romero-Sánchez, M., Krahé, B., Moya, M., \& Megías, J. L. (2017). Alcohol-related victim behavior and rape myth acceptance as predictors of victim blame in sexual assault cases. Violence 
Against Women, 24(9), 1052-1069. https://doi. org//0.I I77//07780I2I 7727372

Rominski, S. D., Darteh, E., \& Munro Kramer, M. (2016). Rape myth acceptance among students at the University of Cape Coast, Ghana. International Journal of Gynecology \& Obstetrics, 136(2), 240-24I. https://doi. org/I0.1002/ijgo. 12054

Rubinsky, V., Hosek, A. M., \& Hudak, N. (20I9). "It's better to be depressed skinny than happy fat": college women's memorable body messages and their impact on body image, selfesteem, and rape myth acceptance. Health Communication, 34(I3), I555-1563. https:// doi.org/I 0.1080/I04I0236.20I8.I504659

Saldívar, G., Jiménez, A. J., Gutiérrez, R., \& Romero, M. (20I5). La coerción sexual asociada con los mitos de violación y las actitudes sexuales en estudiantes universitarios. Salud Mental, 38(I), 27-32. https://doi.org/I0.177I I/ SM.0185-3325.2015.003

Saldívar, G., Ramos, L., \& Saltijeral, M. (2004). Validación de las escalas de aceptación de la violencia y de los mitos de violación en estudiantes universitarios. Salud Mental, 27(6), 40-49.

Saucier, D. A., Strain, M. L., Hockett, J. M., \& McManus, J. L. (20/5). Stereotypic beliefs about masculine honor are associated with perceptions of rape and women who have been raped. Social Psychology, 46, 228-24I. https://doi.org// 0.1027//864-9335/a000240

Schulze, C., \& Koon-Magnin, S. (2017). Gender, sexual orientation, and rape myth acceptance: preliminary findings from a sample of primarily LGBQ-identified survey respondents. Violence and Victims, 32(I), I59-180. https:// doi.org//0.189I/0886-6708.vv-d-15-000I7

Schuster, I., Krahé, B., llabaca, P., \& Muñoz-Reyes, J. A. (2016). Sexual aggression victimization and perpetration among male and female college students in Chile. Frontiers in Psychology, 7, 1354. https://doi.org//0.3389/ fpsyg.2016.01354

Silver, N., \& Hovick, S. R. (2018). A schema of denial: the influence of rape myth acceptance on beliefs, attitudes, and processing of affirmative consent campaign messages. Journal of Health Communication, 23(6), 505-5/3. https://doi.or g/I0.1080/I08I0730.20I8.1473532

Sleath, E., \& Bull, R. (2015). A brief report on rape myth acceptance: differences between police officers, law students, and psychology students in the United Kingdom. Violence and Victims, 30(I), 136-I47. http://doi. org/|0.189|/0886-6708.VV-D-13-00035
Smith, M., Wilkes, N., \& Bouffard, L. A. (2015). Rape myth adherence among campus law enforcement officers. Criminal Justice and Behavior, 43(4), 539-556. https://doi. org//0.1 I77/00938548/5604/78

Stephens, T.,Kamimura,A.,Yamawaki,N.,Bhattacharya, H., Mo, W., Birkholz, R., Makomenaw, A., \& Olson, L. (20I6). Rape myth acceptance among college students in the United States, Japan, and India. SAGE Open, 6(4). https://doi. org/I0.I I77/2I 582440I66750I5

Stoll, L. C., Lilley, T. G. \& Block, R. (2018). The effects of gender-blind sexism on rape myth acceptance: results from a nationally representative study. Journal of Interpersonal Violence, 36(II-I2), 5838-5859. https://doi. org/ $\mid 0.1$ I $77 / 08862605|88079| 2$

Struckman-Johnson, D., \& Struckman-Johnson, C. (1995). College men's reactions to hypothetical forceful sexual advances from women. Journal of Psychology \& Human Sexuality, 8(I-2), 93-105. https://doi. org/I0.1300/J056v08n0I_07

Süssenbach, P., Bohner, G., \& Eyssel, F. (20/2). Schematic influences of rape myth acceptance on visual information processing: an eyetracking approach. Journal of Experimental Social Psychology, 48(3), 660-668. https://doi. org/I0.10I6/j.jesp.20II.12.005

Süssenbach, P., Eyssel, F., \& Bohner, G. (20|3). Metacognitive aspects of rape myths: subjective strength of rape myth acceptance moderates its effects on information processing and behavioral intentions. Journal of Interpersonal Violence, 28(II), 2250-2272. https://doi.org//0.1 I77/08862605 I2475317

Süssenbach, P., Eyssel, F., Rees, J., \& Bohner, G. (20I5). Looking for blame: rape myth acceptance and attention to victim and perpetrator. Journal of Interpersonal Violence, 32(15), 2323-2344. https://doi.org//0.1 I77/08862605/559/975

Tal-Or, N., \& Tsfati, Y. (2018). Does the coviewing of sexual material affect rape myth acceptance? The role of the co-viewer's reactions and gender. Communication Research, 45(4), 577-602. https://doi. org/I 0.1 I 77/00936502/5595073

Taschler, M., \& West, K. (2016). Contact with counter-stereotypical women predicts less sexism, less rape myth acceptance, less intention to rape (in men) and less projected enjoyment of rape (in women). Sex Roles, 76(7-8), 473-484. https://doi.org//0.1007/ sll199-016-0679-x

Thompson, B. A., \& Ortiz, R. R. (2016). Frat daddies and sorostitutes. Sexualization, Media \& Society, 2(4). https://doi. org/I0.II77/23746238I668469| 
Ullman, S. E. (2003). A critical review of field studies on the link of alcohol and adult sexual assault in women. Aggression and Violent Behavior, 8(5), 47I-486. https://doi.org//0.1016/s I359I 789(03)00032-6

Unidad Fiscal Especializada en Violencia Contra las Mujeres. (2019). Relevamiento de fuentes secundarias de datos sobre violencia sexual a nivel país y en la Ciudad Autónoma de Buenos Aires. UFEM. https://www.mpf.gob. ar/ufem/files/2019/10/Informe_UFEM.pdf

Vance, K., Sutter, M., Perrin, P. B., \& Heesacker, M. (20I5). The media's sexual objectification of women, rape myth acceptance, and interpersonal violence. Journal of Aggression, Maltreatment \& Trauma, 24(5), 569-587. https://doi.org/10.1080/10926771.2015.1029 179

Venema, R. M. (20I6). Making judgments: how blame mediates the influence of rape myth acceptance in police response to sexual assault. Journal of Interpersonal Violence, 34(I3), 2697-2722. https://doi.org/I0.I I77/08862605 I 6662437

Vonderhaar, R. L., \& Carmody, D. C. (2014). There are no "innocent victims" the influence of just world beliefs and prior victimization on rape myth acceptance. Journal of Interpersonal Violence, 30(10), 1615-1632. https://doi. org/ I0.I I77/08862605 I4549|96

Walfield,S.M.(20I8). “Mencannotberaped”: correlates of male rape myth acceptance. Journal of Interpersonal Violence, 6(13-14), 639|-6417. https://doi.org/ I0. I 177/08862605 I 881 7777

Ward, C. (1988). The attitudes toward rape victims scale. Psychology of Women Quarterly, I2(2), I27-I46. https://doi. org/| 0. I I I / j. |47|-6402.1988.tb00932.x
Watson, L. C. (2016). Exploring the psychometric properties of the acceptance of modern myths about sexual aggression (AMMSA) scale. Electronic Theses and Dissertations, I 339. https://digitalcommons.georgiasouthern.edu/ etd/I 339

Willis, M., Birthrong, A., King, J. S., Nelson-Gray, R. O., \& Latzman, R. D. (2017). Are infidelity tolerance and rape myth acceptance related constructs? An association moderated by psychopathy and narcissism. Personality and Individual Differences, I I 7, 230-235. https:// doi.org/10.1016/j.paid.2017.06.015

Wilson, L. C., Newins, A. R., \& White, S. W. (2017). The impact of rape acknowledgment on survivor outcomes: the moderating effects of rape myth acceptance. Journal of Clinical Psychology, 74(6), 926-939. https://doi. org/ I0.1002/jclp.22556

Worthen, M. G. F. (2017). Rape myth acceptance among lesbian, gay, bisexual, and mostly heterosexual college students. Journal of Interpersonal Violence, 36( I-2), NP232-NP262. https://doi.org//0.1 I77/08862605 I7733282

Zinzow, H. M., Thompson, M. P., Goree, J., Fulmer, C. B., Greene, C., \& Watts, H. A. (20/8). Evaluation of a college sexual violence prevention program focused on education, bystander intervention, and alcohol risk reduction. College Student Affairs Journal, 36(2), II0-125. https://doi. org/10.1353/csj.2018.0019 